\title{
La finalización de las posesiones en baloncesto: estudio de la acción de finalización
}

\section{The completion of the possessions in basketball: study of the completion action}

\author{
Iria Uxía Romarís ${ }^{1}$ Ignacio Refoyo² y Javier Coterón ${ }^{2}$.
}

${ }^{1}$ Universidade da Coruña, ${ }^{2}$ Universidad Politécnica de Madrid

PALABRAS CLAVE: Baloncesto, ataque, finalización, acción de finalización RESUMEN: En esta investigación se analiza la finalización del ataque desde la perspectiva táctica, estudiando la acción de finalización. Utilizando la metodología observacional, se analizan 4605 posesiones de Liga ACB y Liga Femenina de la temporada 2009/10; con los objetivos de estudiar la finalización de las posesiones en Liga ACB y Liga Femenina, para conocer las acciones de finalización más habituales y estudiar su asociación con el tipo de ataque, la zona desde la que se finaliza y el resultado de la posesión. Haciendo un análisis general de los datos, se observa que aproximadamente la mitad de los ataques finalizan a partir de una ventaja generada de una jugada individual o de un bloqueo directo $(27,8 \%$ y $28,7 \%$ de las posesiones de Liga ACB analizadas y $28,9 \%$ y $17,5 \%$ de las de Liga Femenina). En un análisis pormenorizado, se aprecian diferencias estadísticamente significativas en el uso de las acciones de finalización en Liga ACB y Liga Femenina (Chi-cuadrado para una muestra $\mathrm{p}=0,000$ ), a pesar de que en las dos subpoblaciones la jugada individual exterior es la acción más utilizada y una de las que tienen mejores resultados. En ACB predominan las finalizaciones con bloqueo directo, con buenos porcentajes de eficacia; en Liga Femenina los movimientos sin balón, la circulación de balón y la jugada individual exterior e interior son las acciones más propicias y efectivas para la finalización. Aunque las finalizaciones de los ataques posicionales parten de acciones variadas, las de los contraataques y transiciones están condicionadas por el tipo de ataque al que pertenecen; además la acción de finalización está asociada a la zona de finalización y ligeramente al resultado.
KEYWORDS: Basketball, offence, completion, completion action SUMARY: In this research offence's completion is analyzed from the tactic perspective, studying the completion action. Using observational methodology, 4605 possessions are analysed from Spanish Professional League (ACB League) and Women's Spanish League of the 2009/10 season; with the aim of studying the possessions' completion of ACB and Women's League, to meet the most common completion actions and study them association with the offence's type, the completion area and possession's result. In a general data analysis, it notes that approximately half of the offences end from an advantage generated by an individual move or a direct screen (27.8\% and $28.7 \%$ of the ACB League's possessions analyzed and $28.9 \%$ and $17.5 \%$ of the Women's League ones). In a detailed analysis, statistically significant differences are detected in completion actions's use between ACB League and Women's League (one-sample Chi-square p=0.000), although outside individual move is the most used action and one of those have best results in both samples. The completions using direct screen predominate in ACB and they also have good efficacy percentages; whereas in Women's League movements without ball, ball circulation and outside and inside individual move are the most favourable and effective actions for the completion. Although set offence's completions start with various actions, fast break and transition's ones are conditioned by the type of offence they belong; furthermore completion action is associated with the completion area and slightly associated with the result.

\section{Introducción}

La finalización de la posesión, como fase determinante en el resultado del ataque, ha sido muy estudiada. Desde el punto de vista técnico el análisis se centra en los tipos de lanzamiento (Cárdenas, Moreno y Almendral, 1995; Refoyo, Romarís y Sampedro, 2009; Vieira, 2010). Desde el punto de vista estadístico se valoran las zonas de finalización y los porcentajes de eficacia, existe una prolífera línea de investigación centrada en el análisis de los datos estadísticos de los partidos en busca de los indicadores del éxito o del rendimiento (Brandao, Janeira y Sampaio, 2002; Gómez y Lorenzo, 2005; Karipidis, Fotinakis, Taxildaris y Fatouros, 2001; Leite, 2003;

Dirección de contacto: Iria Uxía Romarís Durán

E-mail: iria_uxia@yahoo.es

Este trabajo ha sido financiado por el programa de recursos humanos María Barbeito (nueva adjudicación del año 2008 y prórroga de 2010) de la Dirección Xeral de Investigación, Desenvolvemento e Inovación de la Xunta de Galicia, cofinanciado con el Fondo Social Europeo.
Leite y Sampaio, 2006; Sampaio, 1998). No existen tantas investigaciones que hayan analizado la finalización de la posesión desde un punto de vista táctico. Desde esta perspectiva, Remmert (2003) señala a los bloqueos directos como las acciones más utilizadas para finalizar los ataques; los bloqueos consecutivos, pasar y cortar y los bloqueos indirectos son, por este orden, los medios tácticos colectivos básicos con los que se consiguen más puntos por ataque. En contraposición con estos resultados Pinto (1999) apunta a las salidas de los bloqueos indirectos como una de las acciones menos eficaces, junto con el 1x1 en el poste bajo; y afirma que las acciones más ligadas al éxito son los cortes laterales que permiten situaciones de $1 \times 1$ y $2 \times 1$.

En esta investigación se analiza la finalización de la posesión desde la perspectiva táctica, con los objetivos de estudiar la finalización de las posesiones en Liga ACB y Liga Femenina, conocer qué acciones de finalización son más habituales y analizar la posible asociación de la acción de finalización con 
el tipo de ataque, la zona desde la que se finaliza y el resultado de la posesión. Para ello se define la acción de finalización como aquella acción o medio táctico colectivo, por el que se genera la ventaja que da lugar a la finalización del ataque con un lanzamiento a canasta, o aquella de la que se intenta aprovechar la ventaja creada o con la que se intenta generar ventaja, cuando se finaliza el ataque con la seńalización de una falta, infracción, balón enviado fuera del terreno de juego o pérdida de la posesión del balón.

Alarcón (2008, p. 514) define la ventaja como "el desequilibrio creado al adversario que defiende al jugador con balón, durante la realización del medio (puede ser cualquiera de los oponentes que participen en el medio) y que le permite lanzar o avanzar hacia el cesto sin oposición". Se considera que un atacante tiene ventaja cuando se encuentra en alguna de las siguientes situaciones (adaptado de Alarcón, 2008): ventaja posicional relativa o máxima (el defensor más cercano al jugador con balón se encuentra sólo con parte de su cuerpo en la línea imaginaria que une al jugador con balón con el aro, o no está en esa línea), ventaja por distancia (el defensor está a una distancia del jugador con balón suficiente para que éste último pueda tirar con comodidad, cuando el atacante con balón se encuentre en una posición desde la que pueda lanzar con eficacia), ventaja por orientación (el defensor no está orientado hacia el jugador con balón), ventaja por cercanía al cesto (a pesar de tener un defensor cercano y bien colocado, un atacante puede ganar la posición para recibir dentro de la zona y si recibe puede jugar eficazmente al estar cerca del aro, por tener una clara superioridad física sobre el defensor, fruto de un cambio de emparejamiento defensivo).

\section{Método}

Utilizando la metodología observacional (Anguera, 1983) se analizaron las 4605 posesiones de la muestra de 14 partidos de Liga ACB y 12 de Liga Femenina de la temporada 2009/10. La recogida de datos se llevó a cabo mediante la observación sistemática y natural de las grabaciones de los partidos y el análisis estadístico de los datos se efectuó con el programa SPSS v. 11.5, realizando estudios descriptivos y correlacionales de las variables.

\section{Resultados}

Analizando las acciones de finalización más habituales en liga ACB y Liga Femenina, se observa que aproximadamente la mitad de los ataques finalizan a partir de una ventaja generada de una jugada individual o de un bloqueo directo, representando el $27,8 \%$ y $28,7 \%$ respectivamente de todas las posesiones de Liga ACB analizadas y el $28,9 \%$ y $17,5 \%$ de las de Liga Femenina. A éstas le sigue el bloqueo indirecto y la categoría "otra acción" (que engloba los ataques en los que la ventaja para la finalización surge de la situación de juego, no de una acción, como en el caso de los contraataques).

Fruto de estas acciones se puede generar una finalización directa, realizada por un jugador involucrado en la acción de finalización, o se puede producir una fijación defensiva que sea aprovechada por medio de pases y finaliza el ataque un jugador no implicado en la acción de finalización, favorecido por la ventaja creada anteriormente. El 69,5\% de las posesiones de la muestra de liga ACB y el 72,2\% de Liga Femenina finalizan de forma directa. Además de ser más numerosas, las finalizaciones directas resultan más eficaces que las finalizaciones por fijación defensiva, sin embargo la diferencia entre ellas no es suficiente como para afirmar que este factor sea determinante en el rendimiento de las finalizaciones (Coeficiente Phi: -0,063 Liga ACB / -0,078 Liga Femenina).

En la tabla 1 se muestran de forma pormenorizada los resultados de la investigación, definiendo más las acciones de finalización y combinándolas con las opciones de finalización (directa o fijación defensiva). 
TABLA 1. Resultados del uso y eficacia de las acciones de finalización en Liga ACB y Liga Femenina

\begin{tabular}{|c|c|c|c|c|c|c|c|}
\hline & & \multicolumn{3}{|c|}{ LIGA ACB } & \multicolumn{3}{|c|}{ LIGA FEMENINA } \\
\hline & & $\mathrm{N}$ & \%Vál & \%Eficacia & $\mathrm{N}$ & \%Vál & \%Eficacia \\
\hline \multirow{24}{*}{ Válidos } & Movimientos sin balón & 109 & $4,9 \%$ & $47,7 \%$ & 181 & $9,2 \%$ & $54,7 \%$ \\
\hline & Movimientos sin balón y pase & 23 & $1,0 \%$ & $52,2 \%$ & 31 & $1,6 \%$ & $32,3 \%$ \\
\hline & Circulación de balón & 95 & $4,3 \%$ & $47,4 \%$ & 177 & $9,0 \%$ & $49,7 \%$ \\
\hline & Circulación de balón y pase & 20 &, $9 \%$ & $45,0 \%$ & 56 & $2,9 \%$ & $39,3 \%$ \\
\hline & Jugada individual interior & 116 & $5,2 \%$ & $45,7 \%$ & 160 & $8,1 \%$ & $53,1 \%$ \\
\hline & Jugada individual interior y pase & 22 & $1,0 \%$ & $31,8 \%$ & 36 & $1,8 \%$ & $36,1 \%$ \\
\hline & Jugada individual exterior & 323 & $14,6 \%$ & $57,0 \%$ & 246 & $12,5 \%$ & $48,4 \%$ \\
\hline & Jugada individual exterior y pase & 154 & $7,0 \%$ & $41,6 \%$ & 126 & $6,4 \%$ & $42,1 \%$ \\
\hline & Bloqueo directo & 206 & $9,3 \%$ & $53,4 \%$ & 138 & $7,0 \%$ & $46,4 \%$ \\
\hline & Bloqueo directo y pase & 202 & $9,1 \%$ & $46,5 \%$ & 98 & $5,0 \%$ & $40,8 \%$ \\
\hline & Bloqueo directo y continuación & 187 & $8,5 \%$ & $51,9 \%$ & 87 & $4,4 \%$ & $46,0 \%$ \\
\hline & Bloqueo directo, continuación y pase & 39 & $1,8 \%$ & $41,0 \%$ & 21 & $1,1 \%$ & $28,6 \%$ \\
\hline & Bloqueo semidirecto & 30 & $1,4 \%$ & $46,7 \%$ & 19 & $1,0 \%$ & $47,4 \%$ \\
\hline & Bloqueo semidirecto y pase & 17 &, $8 \%$ & $52,9 \%$ & 7 &, $4 \%$ & $28,6 \%$ \\
\hline & Bloqueo indirecto & 211 & $9,5 \%$ & $52,1 \%$ & 145 & $7,4 \%$ & $50,7 \%$ \\
\hline & Bloqueo indirecto y pase & 32 & $1,4 \%$ & $50,0 \%$ & 34 & $1,7 \%$ & $32,4 \%$ \\
\hline & Doble bloqueo indirecto & 81 & $3,7 \%$ & $43,2 \%$ & 43 & $2,2 \%$ & $39,5 \%$ \\
\hline & Doble bloqueo indirecto y pase & 31 & $1,4 \%$ & $38,7 \%$ & 23 & $1,2 \%$ & $47,8 \%$ \\
\hline & Bloqueo indirecto doble & 4 &, $2 \%$ & $75,0 \%$ & 10 &, $5 \%$ & $40,0 \%$ \\
\hline & Bloqueo indirecto doble y pase & 4 &, $2 \%$ & $25,0 \%$ & 3 &, $2 \%$ & $0,0 \%$ \\
\hline & Otra acción & 116 & $5,2 \%$ & $67,2 \%$ & 149 & $7,6 \%$ & $58,4 \%$ \\
\hline & Otra acción y pase & 104 & $4,7 \%$ & $55,8 \%$ & 88 & $4,5 \%$ & $58,0 \%$ \\
\hline & Ninguna ventaja & 85 & $3,8 \%$ & $17,6 \%$ & 86 & $4,4 \%$ & $23,3 \%$ \\
\hline & Total & 2211 & $100,0 \%$ & & 1964 & $100,0 \%$ & \\
\hline Perdidos & Sistema & 227 & & & 203 & & \\
\hline Total & & 2438 & & & 2167 & & \\
\hline
\end{tabular}

*\% Vál. = Porcentaje válido $/ \%$ Eficacia $=$ Porcentaje de eficacia

Comparando los resultados detallados de Liga ACB y Liga Femenina se observa que en ambas subpoblaciones es la jugada individual exterior la acción a partir de la que se generan más finalizaciones, a pesar de esta coincidencia existen diferencias estadísticamente significativas en las proporciones del uso de las acciones de finalización entre las dos muestras (Chi-cuadrado para una muestra $\mathrm{p}=0,000$ ). De hecho, si se comparan las siguientes acciones más frecuentemente utilizadas para la finalización, sólo los bloqueos indirectos y los bloqueos directos presentan porcentajes de uso reseñables en las dos subpoblaciones; destacando también en Liga ACB el bloqueo directo y pase y el bloqueo directo y continuación y en Liga Femenina las acciones de finalización: movimientos sin balón, circulación de balón, jugada individual interior y otra acción.
Observando los porcentajes de eficacia mostrados en la tabla 1, se puede advertir la mayor efectividad de las finalizaciones directas con respecto a las finalizaciones por fijación defensiva (pase), que ya se comentó anteriormente. Sólo los movimientos sin balón y el bloqueo semidirecto en Liga ACB y el doble bloqueo indirecto en Liga Femenina obtienen mejores porcentajes de eficacia en la finalización por fijación defensiva.

Hay que resaltar los altos porcentajes de eficacia de la jugada individual exterior, que además de ser la acción con la que más finalizaciones se generan, resulta ser una de las más eficaces. También destacan por sus altos porcentajes de eficacia las finalizaciones a partir de otra acción u otra acción y pase, siendo las que mejores resultados obtienen en Liga Femenina y prácticamente las mejores en ACB. 
En Liga ACB, además de las ya mencionadas, tienen muy buenos resultados las finalizaciones generadas por bloqueo directo, semidirecto y pase, movimientos sin balón y pase, bloqueo indirecto y bloqueo directo y continuación. El bloqueo indirecto doble es la acción de finalización con mejor porcentaje de eficacia (75\%), pero hay que tener cautela con este resultado por tratarse de una acción de la que se analizó una muestra muy pequeña.

En Liga Femenina aparecen otras acciones de finalización con muy buenos porcentajes de eficacia, como son los movimientos sin balón, la jugada individual interior y la circulación de balón, coincidiendo con Liga $\mathrm{ACB}$ en los buenos resultados de las categorías otra acción, otra acción y pase, bloqueo indirecto y jugada individual exterior.

\section{Discusión}

Los resultados generales de esta investigación presentan al bloqueo directo como la acción con la que se generan más finalizaciones en Liga ACB, coincidiendo con los resultados de Remmert (2003) que la señala como la acción más usada para finalizar los ataques; en Liga Femenina es la segunda acción más utilizada. Observando los resultados detallados de ambas categorías (tabla 1), se puede deducir que en Liga ACB existe una mayor dependencia del bloqueo directo, ya que de las cinco acciones más utilizadas tres son opciones de juego del bloqueo directo; sin embargo en Liga Femenina esta acción ocupa el séptimo puesto. Domínguez (2010) en su estudio de los bloqueos directos también destaca su mayor uso en $\mathrm{ACB}$ con respecto a la Liga Femenina. Concuerdan igualmente los resultados de Liga ACB de esta investigación con los de Remmert (2003) al señalar al bloqueo directo como una de las acciones de finalización más eficaces.

No existe unanimidad en los resultados hallados con respecto a los cortes o movimientos sin balón que, salvando las diferencias terminológicas y de categorización de las acciones, Remmert (2003) y Pinto (1999) señalan entre las acciones más eficaces y que en el presente estudio también obtienen muy buenos resultados en Liga Femenina, pero más discretos en ACB; las finalizaciones a partir de bloqueos indirectos están entre las más eficaces del estudio de Remmert (2003) y entre los mejores resultados de esta investigación, sin embargo para Pinto (1999) son de las que peores resultados obtienen; y, por último, la jugada individual interior, una de las menos efectivas en baloncesto masculino (Pinto, 1999; presente estudio), resulta ser una de las más eficaces en la muestra de Liga Femenina.

Los resultados de este estudio muestran una muy leve asociación entre la acción de finalización y la eficacia de la posesión (Coeficiente de Contingencia: 0,181 Liga ACB / 0,171 Liga Femenina); relacionando con un resultado positivo a las finalizaciones en las que la ventaja no surge de las acciones de finalización categorizadas, si no de otras circunstancias del juego (otra acción y otra acción y pase); y con un resultado negativo a los bloqueos directos sólo en Liga Femenina.

Analizando las zonas de finalización se observa una asociación de grado medio con la acción de finalización (Coeficiente de Contingencia: 0,353 Liga ACB / 0,398 Liga Femenina), destacando la ligación entre las finalizaciones de las jugadas individuales interiores y de la categoría otra acción en el interior de la zona y las finalizaciones en el exterior por una circulación de balón. También se asocia, aunque de manera leve (Coeficiente de Contingencia: 0,199 Liga ACB / 0,227 Liga Femenina), la acción de finalización con el lateral en el que se finaliza. En general parecen generar más finalizaciones por el lado derecho los bloqueos directos y por el lado izquierdo los bloqueos indirectos y las jugadas individuales exteriores más pase.

Para terminar, se estudia la influencia del tipo de ataque en la acción con la que finalizan las posesiones, encontrando una fuerte vinculación entre las dos variables (Coeficiente de Contingencia: 0,659 Liga ACB / 0,651 Liga Femenina). Los contraataques se relacionan con finalizaciones en las que la ventaja surge de la situación de juego (otra acción) o se genera con circulaciones de balón o jugadas individuales desde el exterior; las finalizaciones de las transiciones suelen partir de circulaciones de balón, o de llegadas con ventaja que provocan fijación de defensores (otra acción y pase), y en Liga ACB se suman a éstas los bloqueos directos; y las acciones de finalización de los ataques posicionales suelen ser más variadas.

\section{Conclusión}

A continuación se muestran las ideas de mayor relevancia del estudio:

- La jugada individual exterior es la acción de finalización estrella, muy utilizada y con grandes resultados tanto en liga ACB como en Liga Femenina.

- Se deben buscar situaciones de juego claras para conseguir finalizaciones directas a partir de la acción de finalización, ya que la eficacia es menor si se finaliza con pases tras fijaciones defensivas.

- Parece que existen distintos modelos o estilos de juego en baloncesto femenino y masculino, al menos en cuanto a la finalización de los ataques se refiere.

- Ateniéndose a los resultados, en baloncesto masculino deberían buscarse finalizaciones desde bloqueo directo, evitando situaciones de finalización a partir de jugada individual interior.

- En Liga Femenina los resultados apuntan a los movimientos sin balón, la circulación de balón y la jugada individual exterior e interior como las acciones más propicias y eficaces para la finalización; además de las finalizaciones de los contraataques en situaciones de 
ventaja.

- Las acciones de finalización más favorables para lanzar dentro de la zona parecen ser las acciones individuales interiores, sin embargo si interesa finalizar desde el exterior de la línea de 6,25 deberían buscarse situaciones de circulación de balón.

- Aunque las finalizaciones de los ataques posicionales parten de acciones de finalización variadas, las finalizaciones de los contraataques y transiciones están condicionadas por el tipo de ataque al que pertenecen.

\section{Referencias}

Alarcón, F. (2008). Incidencia de un programa de entrenamiento para la mejora táctica colectiva del ataque posicional de un equipo de baloncesto masculino. Granada: Editorial de la Universidad de Granada.

Anguera, Ma T. (1983). Manual práctico de observación. Méjico: Trillas.

Brandao, E., Janeira, M. y Sampaio, J. (2002). 6º Campeonato do Mundo de Juniores Masculinos de Basquetebol: a análise do sucesso realizada a partir das estatísticas do jogo. Lecturas: Educación física y deportes, 45. Recuperado el 10 de Junio de 2008: http://www.efdeportes.com/efd45/ basquet1.htm.

Cárdenas, D., Moreno, M. I. y Almendral, P. (1995). Análisis de los factores que inciden en la eficacia del contraataque en baloncesto. Revista de entrenamiento deportivo, 9 (4), 11-16.

Domínguez, J. (2010). Análisis de la decisión táctica en deportes colectivos. Una aplicación al bloqueo directo en baloncesto. Tesis doctoral no publicada. Universidad Politécnica de Madrid.

Gómez, M. A. y Lorenzo, A. (2005). Diferencias entre equipos ganadores y perdedores en el rendimiento de competición en baloncesto femenino. Kronos, 7, 16-22.

Karipidis, A., Fotinakis, P., Taxildaris, K. y Fatouros, J. (2001). Factors characterising a successful performance in basketball. Journal of Human Movement Studies, 41 (5), 385-397.

Leite, N. M. (2003). Perfil estatístico das equipas da associación de clubs de baloncesto (A.C.B.). II Congreso Ibérico de Baloncesto: la formación y el rendimiento en baloncesto, Cáceres.

Leite, N. y Sampaio, J. (2006). ¿Por qué ganaron o perdieron los partidos de baloncesto los equipos que participaron en el Eurobasket 2005?. Kronos, 9, 67-73.

Pinto, A. J. (1999). Acçoes técnico-tácticas que conduzem á conversao de pontos nos jogos de basquetebol. Monografía de Licenciatura, UTAD.

Refoyo, I., Romarís, I. U. y Sampedro, J. (2009). Analysis of men's and women's basketball fast-breaks. Revista de Psicología del Deporte, 18 (Suppl.), 439-444.

Remmert, H. (2003). Analysis of group-tactical offensive behaviour in elite basketball on the basis of a process orientated model. European Journal of Sports Science. 3 (3), 1-12.

Vieira, I. M. (2010). O contra-ataque em basquetebol. Estudo descritivo e comparativo no escalao sub-16 feminino e masculino da Associaçao de basquetebol do Porto. Porto: Universidade do Porto. 
